Ann. Zootech., Ig6r, 10 (2), 89-96

\title{
LE DOSAGE DU COBALT DANS LES VÉGÉTAUX
}

\author{
J. MAGNY, P. CABROL et J. CARLFS \\ Ecole supérieure d'Agriculture de Purpan, \\ Laboratoire de Physiologie végétale de l'Institut catholique de Toulouse.
}

\section{SOMMAIRE}

La teneur en cobalt des végétaux se situant aux environs de o,I mg/kg de poids sec, une méthode d'ultramicrodosage s'impose. L'extraction est faite à partir d'une solution chlorhydrique des cendres par un réactif spécifique du cobalt, l' $\alpha$-nitroso- $\beta$-naphtol ; pour éliminer les ions gênants, le complexe formé est extrait par le chloroforme. Une nouvelle minéralisation de cet extrait permet un dosage colorimétrique avec le sel nitroso-R. La sensibilité de cette méthode allant au delà du dixième de $\gamma$ par ml, le dosage devient possible sur un échantillon végétal de l'ordre de $20 \mathrm{~g}$.

\section{Importance du cobalt}

Le cobalt est si peu abondant dans les tissus animaux que l'on a soupconné depuis fort peu de temps son importance et sa nécessité vitale.

Cependant, dès 1807 , J. HoGg remarquait une certaine anémie des bovins et des ovins caractérisée par de la léthargie, une perte de poids, une accélération du pouls et de la respiration, la perte de lait, l'avortement, etc... qui paraissaient liés à la fréquentation de certains pâturages et qui disparaissaient sitôt qu'on menait paître ailleurs.

En I925, G. Bertrand et Machebcur, après d'assez nombreux dosages du cobalt, commencèrent à soupçonner son importance dans la vie animale. Cette importance fut nettement confirmée par la découverte en rg49 des cobalamines et de la vitamine $B$ I2.

En I935, Filmer et UNDERWOOD découvraient en Australie que la maladie signalée depuis plus de cent ans sous les noms les plus divers (maladie de la brousse, mal du Danemark, maladie du sel, etc...) était due à une carence en cobalt. Depuis lors les travaux se sont multipliés.

Les sols contiennent en général de I à $40 \mathrm{mg}$ de cobalt par $\mathrm{kg}$ : STEWART parle cependant de I à $300 \mathrm{mg}$ et PATTERSON 0,5 à 2IOO, mais il s'agit ici de la totalité du cobalt présent. Extrait à l'acide acétique ou chlorhydrique, le taux descend très souvent au dessous de o, I et même de o,oI mg (Swaine). 
Les carences apparaissent chez les animaux qui pâturent sur des sols possédant moins de $5 \mathrm{mg}$, car le taux de cobalt de ces pâturages tombe au dessous de $0,07 \mathrm{p}$. p. m. soit $70 \gamma$ par $\mathrm{kg}$ de matière sèche (MONieR, WilliaMs) ; pour PEYve ce taux critique du cobalt du sol serait de $2 \mathrm{mg}$.

Les animaux ont des besoins précis en cobalt, et ces besoins sont deux fois plus importants pendant leur jeunesse et leur période de croissance. La quantité journalière minimale pour un bovin adulte est d'environ I mg par jour, $0, I$ pour un ovin (BEESON). Etant donné la quantité moyenne de fourrage ingéré, ce minimum ne peut être atteint lorsque le taux de cobalt descend au-dessous de $70 \gamma$ par kg de matière sèche.

On remédie à cette carence d'une manière immédiate en fournissant des sels de cobalt dans la boisson ( $2 \mathrm{mg}$ de cobalt par jour, ou $9 \mathrm{mg}$ de sulfate de cobalt, suffsent à guérir un bovin), soit d'une manière lointaine et préventive en fournissant au sol des engrais enrichis en cobalt $(2,5 \mathrm{~kg}$ de sulfure ou de chlorure de cobalt par hectare et par an (RIEHM).

\section{Le dosage du cobalt}

Difficultés du dosage.

La principale difficulté de dosage du cobalt résulte de son peu d'abondance et de la nécessité où l'on se trouve cle traiter de volumineux échantillons, ce qui mu1tiplie les possibilités d'erreurs ou de contaminations. Pour diminuer l'importance de ces prélèvements, il est indispensable de trouver des méthodes de plus en plus précises de microdosages et même d'ultramicrodosages, capables d'apprécier des quantités de l'ordre du $Y$ : on pourra ainsi pour l'échantillonnage, descendre au dessous de roo grammes de poids sec.

Dans ces conditions, beaucoup de méthodes gravimétriques, volumétriques ou même spectrographiques, deviennent pratiquement inutilisables. Parmi les méthodes colorimétriques, celles au thyocyanate ou même à l' $\alpha$-nitroso- $\beta$-naphtol sont encore trop peu sensibles; seul le sel nitroso-R permet l'ultramicrodosage puisqu'il parvient à déceler un vingtième de $\gamma$ de cobalt par ml. C'est en I92I que $\mathrm{H}$. S. VAN KLOOSTER découvrit la remarquable propriété de ce sel dont la solution aqueuse jaune devient rouge an présence de cobalt. Ce sel est le dérivé distulfonique de 1' $\alpha$-nitroso- $\beta$-naphtol.

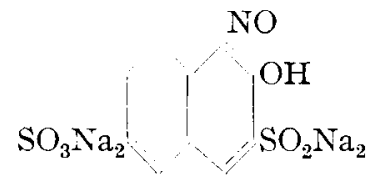

Une trentaine de méthodes de dosage du cobalt dans divers milieux par le sel nitroso-R. ont été publiées. La principale difficulté rencontrée provient des interférences provoquées par des cathions mille fois plus abondants dans la plante que le cobalt, et dont il est difficile de se débarrasser. La difficulté est en grande partie résolue par un néthode telle que celle mise au point par SaLTzMan pour le dosage du cobalt dans les viandes en I955: il utilise 1' $\alpha$-nitroso- $\beta$-naphtol, réactif colorimétrique du cobalt, non pour le doser, mais pour le faire passer dans un composé organique soluble dans le chloroforme qui permet ainsi d'extraire le cobalt à pett près seul et de le doser par le sel nitroso-R. 
La méthode que nous avons mise au point pour le dosage du cobalt dans les végétaux, utilise ce même principe et comporte quelques améliorations de détail.

\section{MATÉRIEL E'T MÉTHODES}

\section{Prinipe.}

On dose colorimétriquement le sel nitroso-R. du cobalt dont la combinaison avec $\mathrm{l}^{\circ} \alpha$-nitroso$\beta$-niaphtol a été extraite par un mélange de chloroforme et d'alcool amylique.

\section{Réactifs nécessaires.}

I - Deux solutions aqueuses de citrate de sodium (environ $300 \mathrm{~g}$ par litre) de pH 8,5 et de pH 5,2: le pH est ajusté par un peu d'acide citrique ou de soude.

2 - Solution de $\alpha$-nitroso- $\beta$-naphtol à 2 p. Ioo dans lacide acétique pur.

3 - Réactif nitroso-R. : pour Ioo $\mathrm{ml}$, on ajoute à $70 \mathrm{ml}$ d'eau $20 \mathrm{ml}$ de citrate de sodium à pH 8,5, puis Io $\mathrm{ml}$ de sel nitruso-R. à $2 \mathrm{p}$. 100 dans l'eau. Ce réactif se conserve facilement 8 jours à l'obscurité.

\section{Mode opératoire.}

Le végétal placé dans une capsule en porcelaine est calciné d'abord sur plaque chauffante, puis au four a moufle à $75^{\circ} \mathrm{C}$ pendant 3 heures. I.es cendres sont reprises par quelques gouttes de $\mathrm{HCl}$ concentré que l'on évapore à sec sur plaque chauffante, puis 3 fois successivement par $\mathrm{HCl}$ I/4, bouillant, et on filtre pour éliminer l'insoluble. Le filtrat est alors évaporé sur bain de sable dont la température ne dépasse pas $80^{\circ} \mathrm{C}$. Le résidu sec est repris par Io $\mathrm{ml}$ de $\mathrm{HCl}$ normal et porté à l'ébullition pour bien dissoudre le précipité.

Pour extraire le cobalt, on ajoute dans le liquide refroidi $70 \mathrm{ml}$ d'eau, $20 \mathrm{ml}$ de citrate à $\mathrm{pH} 5,2$ et I ml de la solution d' $\alpha$-nitroso- $\beta$-naphtol, on agite plusieurs fois le flacon pour homogénéiser le liquide.

Au bout d'une heure environ, on introduit le liquide, en ajoutant l'eau des rinçages, dans une ampoule à décantation de $250 \mathrm{ml}$. Après l'addition de $5 \mathrm{ml}$ d'alcool amylique, qui facilite la séparation, et de $15 \mathrm{ml}$ de chloroforme, on agite vigoureusement lampoule pendant 3 minutes. Le liquide surnageant perd toute la coloration rouge sombre du composé du cobalt avec l' $\alpha$-nitroso- $\beta$-naphtol qui se concentre dans le chloroforme que l'on décante.

Ia solution est évaporée sur bain de sable, au dessous de $50^{\circ} \mathrm{C}$ pour éviter les pertes par projection. Par une attaque nitroso-perchlorique (5 d'acide nitrique pour 2 d'acide perchlorique), l' $\alpha$ nitroso- $\beta$-naphtol est détruit sur plaque chauffante dans un bécher recouvert d'un verre de montre, et le liquide redevenu limpide est évaporé à sec.

Pour doser le cobalt, on dissont le résidu sec dans ro $\mathrm{ml}$ de réactif nitroso-R. et l'on verse la solution dans un tube à essai qui sera placé dans un bain-marie à température constante de $90^{\circ} \mathrm{C}$. Après ro minutes, pour détruire l'excès de réactif et les complexes des métaux qui peuvent encore subsister, on ajoute un $\mathrm{ml}$ d'acide nitrique concentré, on agite vigoureusement le tube pendant 20 secondes et on le remet au bain-marie pendant ro minutes.

Il est alors refroidi rapidement et passé après 15 minutes au colorimètre avec un filtre à 430 mu.

La sensibilité descend au dessous de $\mathrm{I} / \mathrm{I}^{\mathrm{e}}$ de $\gamma$ par $\mathrm{ml}$, puisque ce taux, o, $\gamma / \mathrm{ml}$, donne une déviation de ro graduations à l'électrophotomètre Meunier. Il est facile de construire une courbe pour les dosages, car la loi de Berr est suivie : au moins jusqu'à i $\gamma$ de cobalt par ml, l'intensité de la coloration est proportionnelle à la concentration. Le dosage est précis et fidèle sitôt qu'on a plus de $0,3 \gamma$ par $m l$ de solution, soit $3 \gamma$ dans l'échantillon analysé.

\section{DISCUSSION DES MÉTHODES}

Nous allons examiner quatre problèmes, celui des températures utilisées, celui des interférences possibles, celui des conditions de dosage et celui de l'étalonnage de la courbe. 
I - Les températures utilisées.

Dans un échantillon où 1'on ne dose que le cobalt, il convient de chercher la température qui, sans risque de perte pour cet élément, détruira le mieux les matières organiques.

Cette température peut être portée à $70^{\circ} \mathrm{C}:$ l'incinération est ainsi meilleure qu'à $55^{\circ}$ ou $600^{\circ} \mathrm{C}$ et, non seulement il ne se produit pas de perte de cobalt, mais les dosages comparatifs ont abouti dans ces conditions à des valeurs légèrement plus fortes, d'un centième environ.

Quant au passage au bain-marie où se développe la couleur du dosage, il est indispensable, pour que les données soient comparables entre elles, que la température en soit toujours la même. Cette température qu'il faut essayer d'obtenir la plus élevée possible au dessous de l'ébullition, a toujours été maintenue au voisinage de $90^{\circ} \mathrm{C}\left( \pm \mathrm{I}^{\circ} \mathrm{C}\right)$. Si l'on s'en éloigne, de $5^{\circ} \mathrm{C}$ par exemple, on peut constater dans le dosage une variation susceptible d'entraîner une erreur d'un cinquième de $\gamma$ de cobalt.

Quant aux autres températures que nous utilisons, elles sont moins directement liées au cobalt. La calcination sur plaque chauffante avant le passage au four à moufle est plus progressive et se fait sans risque d'inflammation, car les flammes entraînent souvent des pertes plus ou moins importantes. Pour la même raison, la température du bain de sable doit être telle que les projections qui accompagneraient l'ébullition, soient évitées.

\section{2 - Les interférences.}

Certains éléments sont susceptibles de donner, dans les conditions oit nous nous plaçons, des complexes colorés capables de fausser et de rendre impossible le dosage colorimétrique du cobalt. Parmi ces ions, les plus gênants sont le nickel, le cuivre, le titane, le tungstène et le fer. Pour qu'une solution contenant $\mathbf{I} \gamma$ de cobalt puisse être dosée correctement, il faut qu'il y ait moins de $25 \gamma$ de nickel, moins de Ioo $\gamma$ de cuivre, de titane, de tungstène, et moins de rooo $\gamma$ de fer. Cette éventualité est à craindre lorsqu'on étudie les roches ou les alliages métalliques, mais elle l'est beaucoup moins avec les végétaux où ces ions, relativement peu abondants, sont laissés de côté par le mode d'extraction employé. Pout en être tout à fait sîts, nous avons ajouté à certains échantillons des quantités de fer et de cuivre deux fois plus importantes que les taux maximaux trouvés dans les végétaux : le dosage n'a pas été modifié.

A cette élimination d'influences parasites contribue l'addition, pendant le développement de la coloration, de l'acide nitrique qui détruit les complexes colorés susceptibles de se former et fait disparaître ainsi les couleurs importunes.

\section{3 - Conditions de dosage.}

Une condition importante, que l'on ne doit jamais oublier est la sensibilité à la lumière solaire, de la couleur formée par le sel nitroso-R. avec le cobalt. Voici, par exemple, les variations de lecture au colorimètre pour des échantillons contenant I $\gamma, 2 \gamma, 5 \gamma$ et Io $\gamma$ conservés, après le dosage, comparativement à l'obscurité et au soleil. 


\begin{tabular}{|c|c|c|c|c|c|}
\hline & Témoin & $1 \gamma$ & $2 \gamma$ & $5 \gamma$ & $10 \gamma$ \\
\hline Dosage normal (après $15 \mathrm{mn}$ ). & $122^{\prime}$ & $18^{\prime}-$ & $144^{\prime}$ & 174 & 223 \\
\hline 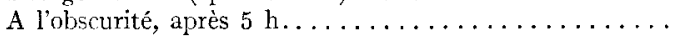 & $12 \cdot 9$ & 132 & 142 & 172 & 221 \\
\hline A l'obscurité, après $30 \mathrm{j} \ldots \ldots \ldots \ldots \ldots \ldots \ldots$ & 63 & 72 & 83 & $11^{\prime}{ }^{\prime}$ & 163 \\
\hline Au soleil, après $24 \mathrm{~h} \ldots \ldots \ldots \ldots \ldots \ldots \ldots \ldots$ & 100 & 91 & & $9 \mathbf{1}^{\prime}$ & 100 \\
\hline
\end{tabular}

Il semble qu'à l'obscurité l'intensité de la couleur diminue lentement, mais cette baisse affecte surtout la coloration du témoin, dont l'intensité a baissé de moitié au bout d'un mois. Cependant, il suffit de considérer ce tableau pour voir que les écarts entre les différentes valeurs se sont à peu près maintenues, tant et si bien qu'après quelques corrections, le dosage est encore possible. Il n'en est pas de même pour les échantillons restés au soleil, et les valeurs obtenules sont ici tout à fait aberrantes. Il est donc important de faire ces dosages à l'abri du soleil.

\section{4 - Etalonnage de la courbe.}

L'intensité de la couleur formée ne varie gutére et ses différentes valeurs sont assez stables pour qu'on puisse construire une échelle de dosage qu'il n'est pas besoin de contrôler. Cependant il convient d'utiliser à chaque dosage un ou deux tubes témoins à blanc pour fournir le zéro de la courbe.

Comme le montre le tableau précédent, jusqu'à Io $\gamma$ dans la prise d'essai, soit $x \gamma / \mathrm{ml}$, la loi de Beer est suivie et l'intensité de la coloration proportionnelle à la concentration.

Ia solution étalon de cobalt se prépare à partir du $\mathrm{CO}_{3} \mathrm{Co}$ préalablement desséché pendant 2 ou 3 heures à I0 $5^{\circ} \mathrm{C}$. Après la pesée, le sel est dissous dans quelques $\mathrm{ml}$ de $\mathrm{HCl}$ dilué bouillant et l'on ajoute suffisamment d'eau pour obtenir une solution de I mg de cobalt par $\mathrm{ml}$ qui servira de solution mère.

\section{RESSULTATS OBTENUS}

Avant de présenter quelques uns des résultats obtenus dans le dosage de Co dans les fourrages, nous voudrions donner quelques précisions sur la fidélité de la méthode et sur les variations de la teneur en cobalt.

\section{I - Fidélité de la méthode.}

Surtout lors des premiers dosages effectués, nous avons toujours pris 2, 3 ou même 4 échantillons du même fourrage pour tester la fidélité de notre méthode. Voici quelques résultats obtenus, sans aucune correction, puisqu'il s'agit des graduations lues au colorimètre pour les dosages comparatifs, alors que le témoin donnait une déviation de I25. Nous avons trouvé, I77-I76-I77, I65-I69-I69, I34-I32, I38-I38, I38-I39, I36-I35, I54-I53, I89-I89, etc...

Puisqu'un dixième de $\gamma$ de cobalt donne une déviation de ro divisions, l'écart entre les lectures représente ordinairement moins de un cinquantième de $\gamma$ de cobalt. Il est évident, d'après ces résultats que ce dosage peut atteindre facilement une précision de $5 \mathrm{p}$. Ioo et même la dépasser lorsque les teneurs sont relativement assez fortes. Cette précision diminue lorsque la quantité de cobalt est trop faible et descend aux environs du dixième de $\gamma / \mathrm{ml}$, soit I $\gamma$ dans l'ensemble de l'échantillon étudié. 
Des expériences de surcharge en cobalt ont été faites pour tester encore la méthode. Des quantités variant de I à ro ont été ajoutées systématiquement à un même échantillon de fourrage : ces quantités ont toujours été exactement retrouvées avec la précision que nous venons de décrire.

\section{2 -Variations $d u$ cobalt.}

Bien que pour un échantillon donné, le dosage du cobalt soit assez fidèle et précis, des échantillons de la même espèce végétale ou bien đu même fourrage pris en des endroits différents du champ peuvent varier dans d'assez fortes proportions. Ces variations ne peuvent manquer de surprendre ceux qui n'ont pas étudié les oligo-éléments : elles rendent nécessaires les moyennes statistiques, si l'on veut se faire de leur teneur une idée tant soit peu précise et qui rende possible les comparaisons.

Pour donner une idée de la variation des oligo-éléments, comparativement avec celle des éléments majeurs, nous allons considérer un ensemble de $8 \mathrm{I}$ dosages de Maïs, cultivés sur des sols différents et représentant 7 variétés différentes : il est donc normal qu'il apparaisse quelques variations dans les teneurs en matières minérales. Nous allons considérer ces teneurs pour deux éléments majeurs, le potassium et le phosphore, et pour deux oligo-éléments, le $\delta$ manganèse et le cobalt, en comparant la moyenne des ro échantillons les plus riches avec celle des io échantillons les plus pauvres en l'élément considéré.

Pour le potassium, les ro échantillons les plus pauvres donnent une moyenne de I,05 p. I0O du poids sec, en face de I,45 pour les plus riches ; pour le phosphore, nous avons $0,175 \mathrm{p}$. Ioo en face de $0,25 \mathrm{p}$. Ioo du poids sec, pour le manganèse, $26 \mathrm{mg} / \mathrm{kg}$ de poids sec en face de $62 \mathrm{mg}$, et pour le cobalt $25 \gamma / \mathrm{kg}$ de poids sec en face de $77 \gamma$.

I a différence entre les deux moyennes, en considérant la plus faible comme égale à $I$, est de $I, 38$ pour le potassium, de $I, 43$ pour le phosphore, mais elle passe à 2,4 pour le manganèse et à 3 , I 5 pour le cobalt. La cohérence des dosages d'oligoéléments est beaucoup moins grande que celle des éléments majeurs.

\section{3 - Premières constatations.}

Elles reposent sur plusieurs centaines de dosages faits sur des fourrages provenant surtout de la région toulousaine, de la Savoie, de l'Aveyron, des Charentes, du pays Basque, etc...

Sur chaque échantillon I 5 éléments ont été dosés, et ces dosages nous ont permis de comparer la teneur en cobalt avec celle de l'ensemble et de chacun des éléments minéraux.

Nous avons remarqué que le cobalt augmente avec l'ensemble des éléments minéraux et que son taux varie avec celui du calcium, du manganèse et du fer : ceci nous ouvre des horizons sur son rôle physiologique trop peu connu, comme nous le montrerons ailleurs. Nous ne retiendrons ici que certains détails intéressants.

La teneur des végétaux en cobalt dépend en partie du terrain et cette teneur augmente si le cobalt abonde dans le sol. Certains sols argilo-calcaires sur marnes des environs de Roquefort ont fourni des fourrages avec des teneurs 2 à 3 fois plus élevées qu'à l'ordinaire.

Cette influence paraît toutefois exceptionnelle. Sur un même sol, la teneur en cobalt varie suivant les espèces végétales et le Maïs par exemple, est beaucoup moins riche que la Betterave. 
Généralement leur taux de cobalt, en même temps que celui de l'ensemble des minéraux, augmente légèrement au cours des coupes successives. Cependant, si le champ est pauvre en cobalt, la teneur, au lieu d'augmenter, diminue progressivement dans les plantes.

Si nous considérons le tableau général des résultats, où nous n'avons retenu des échantillons examinés que les moyennes du cobalt, en $\gamma / \mathrm{kg}$ de matière sèche, et le pourcentage des cendres, nous constatons une certaine diversité.

\begin{tabular}{|c|c|c|c|}
\hline$\ldots$ & $\begin{array}{c}\text { Nombre } \\
\text { d'échantillons }\end{array}$ & $\begin{array}{l}\text { Yatière min. } \\
\text { o du poids sec }\end{array}$ & $\begin{array}{c}\text { Cobalt } \\
\gamma / \mathrm{kg} \mathrm{sec}\end{array}$ \\
\hline $\begin{array}{l}\text { I.égumineuses .................. } \\
\text { (ensemble des échantillons) }\end{array}$ & 52 & 8,05 & $10 \overline{3}$ \\
\hline Luzene................ & 27 & 8,5 & 113 \\
\hline 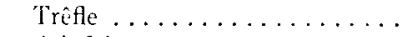 & 10 & 7,65 & $1: 0$ \\
\hline Sianfoin $\ldots \ldots \ldots \ldots \ldots$ & 1:! & 6,7 & 80 \\
\hline $\begin{array}{l}\text { Graminces ..................... } \\
\text { (ensemble des échantillons) }\end{array}$ & 4: & 4,20 & 75 \\
\hline Dartyle $\ldots \ldots \ldots \ldots \ldots$ & 19 & 8,6 & $\pi$ \\
\hline Fétuque $\ldots \ldots \ldots \ldots \ldots$ & 7 & 9,85 & 67 \\
\hline Prairies naturelles ........... & 80 & 6,85 & 87 \\
\hline Prairies artiticielles $\ldots \ldots \ldots \ldots$ & $1: 5$ & 8,9 & 97 \\
\hline Maïs $\ldots \ldots \ldots \ldots \ldots \ldots$ & 81 & 3,6 & 43 \\
\hline Betteraves.................. & 30 & 8,2 & 130 \\
\hline
\end{tabular}

Par strite de la silice qu'elles accumulent, les Graminées sont plus riches en cendres que les Légumineuses, mais celles-ci sont plus riches en cobalt.

Les prairies naturelles, dont la coupe est ordinairement retardée par rapport aux prairies artificielles où les plantes sont récoltées plus jeunes, ont moins de matières minérales par rapport à leur poids sec, mais sont plus riches en cobalt si l'on rapporte celui-ci aux cendres et non au poids sec.

Les Betteraves enfin sont beaucoup plus minéralisées que le Maïs, mais leur richesse en cobalt n'est pas beaucoup plus grande si on la rapporte au poids de cendres. Il n'en reste pas moins vrai que, par rapport au poids sec, qui pour un aliment est tout de même la valeur de base puisqu'elle linite la quantité consommée, la Maïs se situe au dessous du taux critique de $70 \gamma$ de cobalt par $\mathrm{kg}$ de poids sec : il en résulte qu'un animal qui ne recevrait que du Maïs risquerait de souffrir tôt ou tard d'une carence en cobalt.

\section{CONCLUSION}

Bien qu'il demande un certain nombre de précautions, 1'ultramicrodosage du cobalt est relativement facile. Cet élément est extrait de la solution chlorhydrique des cendres par l' $\alpha$-nitroso- $\beta$-naphtol et dosé colorimétriquement, avec une sensibilité supérieure au dixième de $\gamma$, grâce au sel nitroso-R. La plupart des fourrages se situent au-dessus du taux critique pour les animaux de $70 \gamma$ par $\mathrm{kg}$ de poids sec. 


\section{SUMMARY}

the determination of Cobalt in plants.

As the cobalt content of plants is about $0, \mathrm{I} \mathrm{mg} / \mathrm{kg}$ of dry weight, it is necessary to use a method of ultramicrodetermination. The extraction is made from a hydrochloric solution of ash by a specific cobalt reagent : $\alpha$-nitroso- $\beta$-naphtol; in order to eliminate unwanted ions the complex formed is extracted by chloroforme. A new mineralisation of this extract makes it possible to perform a colorimetric determination with nitroso-R. salt. As the sensitivity of this method goes beyond a tenth of $\gamma$ per ml, it is possible to use on a plant sample of about $20 \mathrm{~g}$.

\section{RÉFÉRENCES BIBLIOGRAPHIQUES}

BeEsox K., 1950. Cobalt occurence in soil and forages in relation to a nutritional disorder in ruminants. U. S. Dept. Agric. Washintgon Inform. Bull., 7.

Bertrand G., Machebeup M., I925. Sur les proportions de cobalt contenues dans les organes animaux. C. R. Acad. Sci., 180, 1993.

Filmer J.F., UNDERWoOD E. J., I937. Enzootic marasmus. Further data concerning the potency of cobalt as a curative and prophylactic agent. Aust. Vet. J., 13, 57-64.

Hogg J., I807 (d'après Filmer et UNDERWOOD).

Van KLOOSTER H. S., I921, J. Amer. Chem. Soc., 43, 746.

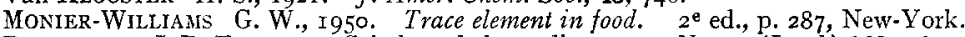

Patterson J. B. F., i937. Cobalt and sheep diseases. Nature (Lond.) 140, 363.

PEYVE J. V., 1956. Recherches pédologiques et agronomiques pour l'emploi difiérencié des engrais. VIo Congr. Sci. sol, 4, 26, I 73-185.

RIEHM H., I954. Kobalt-mangelerscheinungen im Schwartzwald und Wage zu ihrer Heilung. Sonderb. Landa. Forch., 6, I39-I44.

SaltzMAN B. E., KliEMAN R. G., I957. Microdetermination of cobalt in biological materials, in Methods of biochemical analysis. 5, 18 I-223. Interscience New-York.

Stewart J, Mitchell R. L., STEwart A. B., I941. Pining in sheep its control by administration of cobait and by use of cobalt-rich fertilizers. Emp. J. Exp. Agric., 9, 145-I $5^{2}$.

SwaINe D. J., I955. The trace-element content of soils. p. 20.23 Aberdeen. 